\title{
Modeling and Analysis of Multiple Attached Masses Tuning a Piezoelectric Cantilever Beam Resonant Frequency
}

\author{
Yuejuan Li $\mathbb{D}^{1,2}$ Xulei Hou $\mathbb{D}^{1,2}$ Wei Qi $\mathbb{D}^{3},{ }^{1,2}$ Qiubo Wang $\mathbb{D}^{1,2}$ and Xiaolu Zhang $\mathbb{D}^{1,2}$ \\ ${ }^{1}$ College of Mechanical Engineering and Applied Electronics Technology, Beijing University of Technology, Beijing 100124, China \\ ${ }^{2}$ Engineering Research Center of Advanced Manufacturing Technology for Automotive Components, Ministry of Education, \\ Beijing 100124, China \\ ${ }^{3}$ China Automotive Technology \& Research Center Co., Ltd., Tianjin 300300, China
}

Correspondence should be addressed to Xiaolu Zhang; xiaolu.zhang@bjut.edu.cn

Received 3 February 2020; Revised 23 September 2020; Accepted 15 October 2020; Published 27 October 2020

Academic Editor: Sara Amoroso

Copyright (C) 2020 Yuejuan Li et al. This is an open access article distributed under the Creative Commons Attribution License, which permits unrestricted use, distribution, and reproduction in any medium, provided the original work is properly cited.

\begin{abstract}
Mechanical vibrations have been an important sustainable energy source, and piezoelectric cantilevers operating at the resonant frequency are regarded as one of the effective mechanisms for converting vibration energy to electricity. This paper focuses on model and experimental investigations of multiple attached masses on tuning a piezoelectric cantilever resonant frequency. A discrete model is developed to estimate the resonant frequencies' change of a cantilever caused by multiple masses' distribution on it. A mechanism consisted of a piezoelectric cantilever with a $0.3 \mathrm{~g}$ and a $0.6 \mathrm{~g}$ movable mass along it, respectively, is used to verify the accuracy of the proposed model experimentally. And another mechanism including a piezoelectric cantilever with two $0.3 \mathrm{~g}$ attached masses on it is also measured in the designed experiment to verify the discrete model. Meanwhile, the results from the second mechanism were compared with the results from the first one in which the single attached mass is $0.6 \mathrm{~g}$. Two mechanisms have wildly different frequency bandwidths and sensitivities although the total weight of attached masses is the same, $0.6 \mathrm{~g}$. The model and experimental results showed that frequency bandwidth and sensitivity of a piezoelectric cantilever beam can be adjusted effectively by changing the weight, location, and quantity of attached masses.
\end{abstract}

\section{Introduction}

The mechanical vibration is one of the most important sustainable energy sources for several decades and it can be converted to electricity by electrostatic, electromagnetic, and piezoelectric transductions. Among all these conversion mechanisms, piezoelectric transduction is attracting more and more attention because of its higher power density, simpler design, and smaller size [1-4]. Many studies have been done on the applications of piezoelectric materials as energy converters collecting energy from ambient environmental vibrations, such as ocean, buildings, vehicles, etc. [5-9]. Comparing all piezoelectric structural forms, cantilever energy harvesters can collect much energy because cantilever has larger deflection and a larger deflection induces a higher output voltage and power. Therefore, piezoelectric cantilever has been being intensively studied for many years [10]. Many research studies demonstrated that tuning the resonant frequency of the piezoelectric device to match the frequency of vibration sources can increase the efficiency of harvesting energy. To adapt energy converters to any generic applications in many kinds of areas regardless of surrounding conditions, many researchers have tried to find out a variety of approaches to widen the converters' frequency band. Building up piezoelectric cantilever array or bulk, which is consisted of cantilever beams in different sizes with various resonant frequencies and thus can widen the operation bandwidth $[11,12]$, is an effective way to accumulate much energy. Although piezoelectric array can increase the total amount of collecting energy, this is at the expense of the device size and efficiency, because a part of beams will not operate in resonance and hence will not contribute to the harvested energy, which drastically reduces the potential energy density of the device. In order to get 
compact and high harvesting efficiency harvester, many research groups have investigated a variety of methods. Those methods include tryouts of different geometric shapes of piezoelectric cantilevers and active self-adjustable mechanism of resonant frequencies [13-19]. Resonant frequency tuning for piezoelectric cantilever beams through self-adjustable mechanism is regarded as one of the most competent methods. Different methodologies have been developed to adjust the resonant frequencies of piezoelectric devices [10, 20-23]. Among those methods, attaching a proof mass on the cantilever beam is preferred due to the simple structure. By changing the proof mass properties, resonant frequencies (including the first mode, the second mode, and so on) can be adjusted correspondingly. It is also one of the most attractive methods for vibration control whose performance mainly depends on the voltage range [24-26]. Studies have proved that the piezoelectric cantilever harvester operating at the first two resonant frequencies is able to provide high voltage and energy $[11,27]$. Therefore, it is much critical to tune a piezoelectric cantilever working on the first two resonant frequencies through adjusting the attached proof mass. Some research investigated the influence of a mass location on the cantilever frequency and some showed the relationship between a mass weight and cantilever frequency [26-30]. Although plenty of papers have illustrated that an attached mass can tune the frequency wideband of a piezoelectric cantilever, attached multiple masses tuning piezoelectric cantilever resonant frequencies have not been well studied yet. In this paper, a systematic approach will be proposed, and a model will be established to identify frequencies of multimodes for a cantilever beam with multiple movable attaching masses. It provides an efficient and accurate means to estimate the resonant frequencies of a cantilever beam. With this approach, variation of resonant frequencies can be measured when various weight masses move along the cantilever structure. Thus, when fine-tuning of resonant frequency is necessary, engineers can determine proper locations to attach the masses so that the resonant frequencies in different modes can be shifted to desired regions. This simple approach provides a quick reference to evaluate the change of resonant frequencies due to the masses attached on the structure. Theoretical results of the proposed model are verified by experimental tests. The compared results demonstrate a good match between actual measurements and estimated outcomes.

\section{Modelling of Cantilever Beam-Attached Multiple Masses}

2.1. Continuous Model of Cantilever Beam. To model the resonant frequency of a cantilever beam, Rayleigh's method is usually adopted. Generally, the resonant frequency $\omega$ of a cantilever (fixed-free structure) without a proof mass can be expressed in terms of the flexural rigidity EI by [31]

$$
\omega=\frac{v_{n}^{2}}{l^{2}} \sqrt{\frac{\mathrm{EI}}{m^{\prime}}},
$$

where $v_{n}$ is the dimensionless $n$ th-mode eigenvalue, $l$ is the length of cantilever, $E$ is the elasticity modulus, $I$ is the area moment of inertia about the neutral axis, and $m$ ' is the mass per unit length of the cantilever beam. However, this equation can only identify the resonant frequency of a cantilever beam with fixed configuration. If the frequency needs to be changed with an attached mechanism, it is no longer applicable. To change the resonant frequency of a cantilever beam, one easy approach is to add a proof mass at desired locations, which might be difficult to estimate the corresponding fundamental frequency with the above equation. To expand the Rayleigh energy method, the resonant frequency $\omega$ can be obtained with a series of lumped masses. That is,

$$
\omega^{2}=E I \frac{\int_{0}^{l}\left(\mathrm{~d}^{2} y / \mathrm{d} x^{2}\right)^{2} \mathrm{~d} x}{m^{\prime} \int_{0}^{l} y_{1}^{2} \mathrm{~d} x+\Delta m y_{2}^{2}},
$$

where $y$ is the deflection at any point, $x$ is the distance between the point and the end tip of a cantilever, $y_{1}$ is the deflection caused by homogeneous cantilever mass, $\Delta m$ is the mass of lumped mass, and $y_{2}$ is the deflection caused by the attached mass.

Rayleigh's method is highly recommended for deriving fundamental frequencies of uniform cantilever beams with multiple masses at various positions in terms of computational efficiency [32]. Equation (2) demonstrates that the deflection at every point can be critical to determine the resonant frequencies. However, Rayleigh's method can be efficient only if fundamental mode is considered. Once multimodes' vibration needs to be taken into consideration, the advantage of Rayleigh's method will no longer exist since it is difficult to get deflection at all positions. In this case, Newton's second law will be adopted to obtain resonant frequencies of multimodes' vibration.

2.2. Discrete Modelling. Instead of the continuous approach of Rayleigh's method, the cantilever beam can be separated into multiple segments with Newton's second law to derive the equations of motion for individual segments. With this discrete approach, the resonant frequencies can be obtained by lumping all the segments into a matrix representation.

A cantilever is assumed as a uniform and homogeneous beam without any attached mass and a discrete model is started by separating the beam into $n$ segments with equal length. The mass of each segment is concentrated at its midpoint. As an example, a cantilever beam divided into four segments is shown in Figure 1. These four segments are named as $A, B, C$, and $D$ and their corresponding midpoints are called stations $1,2,3$, and 4 . Station 5 is the root of the cantilever beam. The total length of the cantilever is $l$ and the length of each segment is $l / 4$. The mass of the whole cantilever is $m$ and the mass of each segment $\left(m_{1}, m_{2}, m_{3}\right.$, and $\left.m_{4}\right)$ is $m / 4$.

To model a discrete cantilever, the $n$-DOF linear mechanical system by neglecting damping can be written as

$$
\mathbf{m} \ddot{\mathbf{x}}+\mathbf{k x}=\{0\},
$$




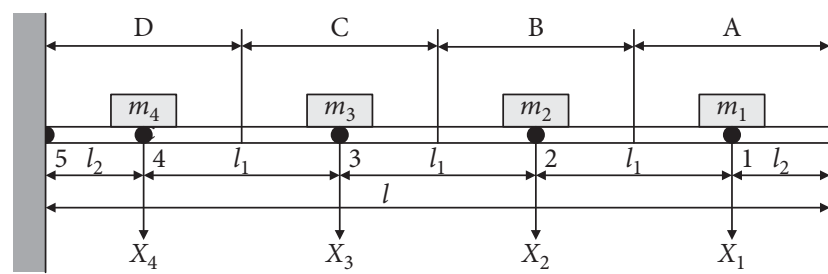

Figure 1: Configuration of a uniform cantilever system.

where $\mathbf{m}$ is the mass matrix, $\mathbf{x}$ is the displacement of every point on the cantilever beam, and $\mathbf{k}$ is the stiffness matrix. Substituting the function of $\mathbf{x}$ with harmonic functions:

$$
\mathbf{x}=\mathbf{A} \sin (\omega t+\phi),
$$

and then multiplying (3) with a matrix $\mathbf{a}=\mathbf{k}^{-1}$, it can be represented as

$$
-\omega^{2} \cdot \mathbf{a} \cdot \mathbf{m} \cdot \mathbf{A}+\mathbf{I} \cdot \mathbf{A}=\{0\} .
$$

Replacing $\lambda$ with $1 / \omega^{2}$, the following equation can be obtained:

$$
(\mathbf{a} \cdot \mathbf{m}-\lambda \mathbf{I}) \cdot \mathbf{A}=\{0\} .
$$

Thus, the characteristic determinant of $(\mathbf{a} \cdot \mathbf{m}-\lambda \mathbf{I})$ becomes

$$
\left|\left[\begin{array}{cccc}
a_{11} & a_{12} & a_{13} & \cdots \\
a_{21} & a_{22} & \cdot & \cdots \\
a_{31} & \cdot & \cdot & \cdots \\
\cdot & \cdot & \cdot & \cdots
\end{array}\right]\left[\begin{array}{cccc}
m_{11} & m_{12} & m_{13} & \cdots \\
m_{21} & m_{22} & \cdot & \cdots \\
m_{31} & \cdot & \cdot & \cdots \\
\cdot & \cdot & . & \ldots
\end{array}\right]-\lambda\left[\begin{array}{cccc}
1 & 0 & 0 & \cdots \\
0 & 1 & 0 & \ldots \\
0 & 0 & 1 & \cdots \\
\cdot & . & . & \ldots
\end{array}\right]\right|=0 .
$$

From (3), the eigenvalues $\lambda_{1}, \lambda_{2}, \ldots, \lambda_{n}$ can be determined, and the frequencies of $n$ modes can be determined as well.

To calculate the resonant frequencies of a system consisted of a cantilever and an attached mass, the mass addition to the cantilever beam is required to be considered as placing a load at one of the stations. A flexibility matrix is obtained through the moment diagrams with the area moment method. The configurations of the system in which the cantilever beam is divided into four segments are shown in Figure 2.

With these configurations, as placing a unit load $P$ at station 1 (see Figure 2(a)), $a_{11}$ is equal to the moment diagram taken at station 1, $a_{21}$ is obtained by the area moment between stations 2 and $5, a_{31}$ is gotten by the area moment between stations 3 and 5 , and $a_{41}$ is gotten by the area moment between stations 4 and 5. The mathematical representation is as follows:

$$
\begin{aligned}
& a_{11}=\frac{1}{2}\left(3 l_{1}+l_{2}\right)^{2} \times \frac{2}{3}\left(3 l_{1}+l_{2}\right) \frac{1}{\mathrm{EI}}=\frac{343}{1536} \frac{l^{3}}{\mathrm{EI}}, \\
& a_{21}=\left[\frac{1}{2} l_{1}\left(2 l_{1}+l_{2}\right) \times \frac{1}{3}\left(2 l_{1}+l_{2}\right)+\frac{1}{2}\left(3 l_{1}+l_{2}\right)\left(2 l_{1}+l_{2}\right) \times \frac{2}{3}\left(2 l_{1}+l_{2}\right)\right] \frac{1}{\mathrm{EI}}=\frac{25}{192} \frac{l^{3}}{\mathrm{EI}}, \\
& a_{31}=\left[\frac{1}{2} \times 2 l_{1}\left(l_{1}+l_{2}\right) \times \frac{1}{3}\left(l_{1}+l_{2}\right)+\frac{1}{2}\left(3 l_{1}+l_{2}\right)\left(l_{1}+l_{2}\right) \times \frac{2}{3}\left(l_{1}+l_{2}\right)\right] \frac{1}{\mathrm{EI}}=\frac{27}{512} \frac{l^{3}}{\mathrm{EI}}, \\
& a_{41}=\left[\frac{1}{2} \times 3 l_{1} l_{2} \times \frac{1}{3} l_{2}+\frac{1}{2}\left(3 l_{1}+l_{2}\right) l_{2} \times \frac{2}{3} l_{2}\right] \frac{1}{\mathrm{EI}}=\frac{5}{768} \frac{l^{3}}{\mathrm{EI}},
\end{aligned}
$$

where $l_{1}=l / 4$ and $l_{2}=l_{1} / 2$. With the same method, $a_{12}, a_{22}, a_{32}$, and $a_{42}$ can be determined by placing a unit load at station 2 (see Figure 2(b)). They are

$$
\begin{aligned}
& a_{12}=\frac{1}{2}\left(2 l_{1}+l_{2}\right)^{2} \times\left[\frac{2}{3}\left(2 l_{1}+l_{2}\right)+l_{1}\right] \frac{1}{\mathrm{EI}}=\frac{25}{192} \frac{l^{3}}{\mathrm{EI}}, \\
& a_{22}=\frac{1}{2}\left(2 l_{1}+l_{2}\right)^{2} \times \frac{2}{3}\left(2 l_{1}+l_{2}\right) \frac{1}{\mathrm{EI}}=\frac{125}{1536} \frac{l^{3}}{\mathrm{EI}}, \\
& a_{32}=\left[\frac{1}{2} l_{1}\left(l_{1}+l_{2}\right) \times \frac{1}{3}\left(l_{1}+l_{2}\right)+\frac{1}{2}\left(2 l_{1}+l_{2}\right)\left(l_{1}+l_{2}\right) \times \frac{2}{3}\left(l_{1}+l_{2}\right)\right] \frac{1}{\mathrm{EI}}=\frac{9}{256} \frac{l^{3}}{\mathrm{EI}}, \\
& a_{42}=\left[\frac{1}{2} \times 2 l_{1} l_{2} \times \frac{1}{3} l_{2}+\frac{1}{2}\left(2 l_{1}+l_{2}\right) l_{2} \times \frac{2}{3} l_{2}\right] \frac{1}{\mathrm{EI}}=\frac{7}{1536} \frac{l^{3}}{\mathrm{EI}} .
\end{aligned}
$$




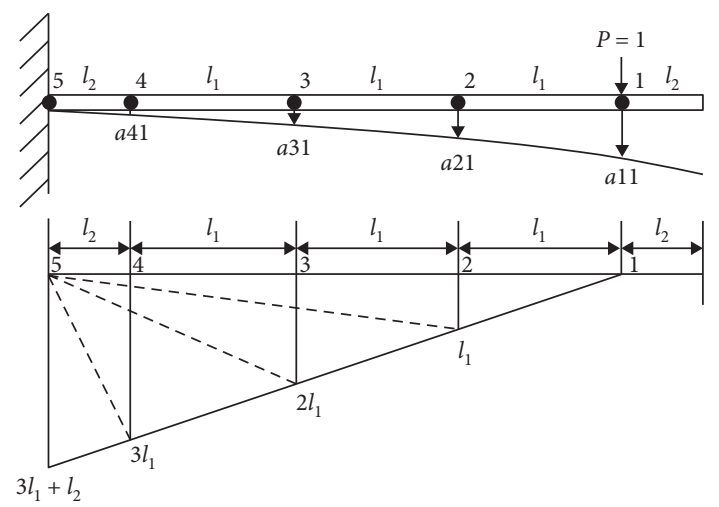

(a)
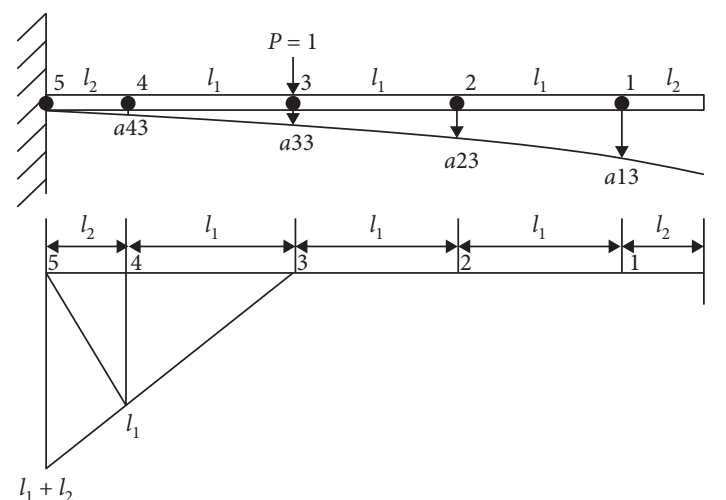

(c)

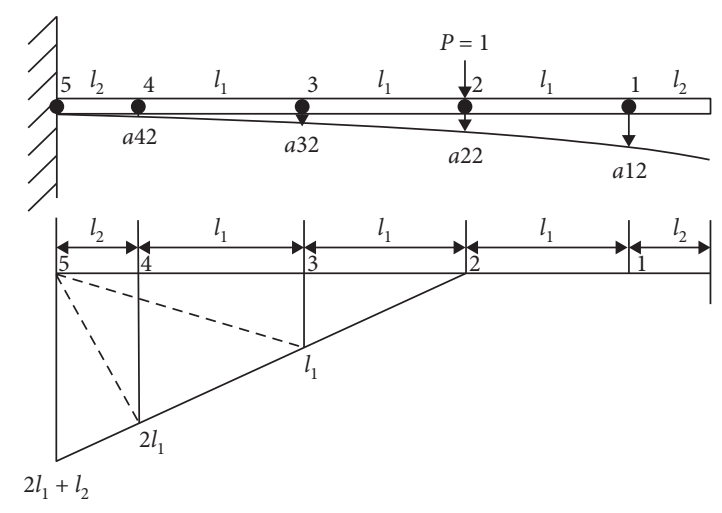

(b)

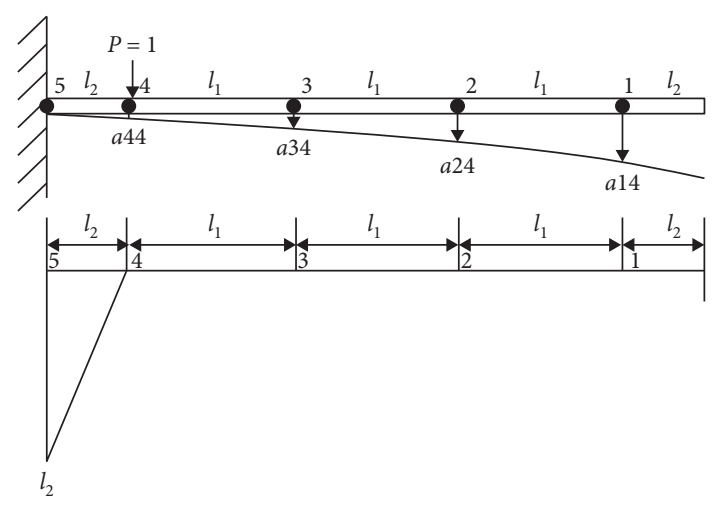

(d)

Figure 2: Moment diagrams of a cantilever beam. (a) Placing a unit load at station 1. (b) Placing a unit load at station 2. (c) Placing a unit load at station 3. (d) Placing a unit load at station 4.

All element values of matrix a can be obtained by repeating the same procedure. Table 1 lists the symbolic representation of the matrix element values if the cantilever is divided into 4 segments.

The cantilever is assumed to be uniform and homogeneous beam, so each segment has the same mass. For the cantilever divided into 4 segments, $m_{1}=m_{2}=m_{3}=m_{4}=m / 4$ and the mass matrix can be written as

$$
\mathbf{m}=\left[\begin{array}{cccc}
m_{1} & 0 & 0 & 0 \\
0 & m_{2} & 0 & 0 \\
0 & 0 & m_{3} & 0 \\
0 & 0 & 0 & m_{4}
\end{array}\right]=\frac{m}{4}\left[\begin{array}{cccc}
1 & 0 & 0 & 0 \\
0 & 1 & 0 & 0 \\
0 & 0 & 1 & 0 \\
0 & 0 & 0 & 1
\end{array}\right] .
$$

TABle 1: Element values of the $4 \times 4$ flexibility matrix a.

\begin{tabular}{lcccc}
\hline$n$ & 1 & 2 & 3 & 4 \\
\hline$a_{1 n}$ & $343 / 1536$ & $25 / 192$ & $27 / 512$ & $5 / 768$ \\
$a_{2 n}$ & $25 / 192$ & $125 / 1536$ & $9 / 256$ & $7 / 1536$ \\
$a_{3 n}$ & $27 / 512$ & $9 / 256$ & $9 / 512$ & $1 / 354$ \\
$a_{4 n}$ & $5 / 768$ & $7 / 1536$ & $1 / 384$ & $1 / 1536$ \\
\hline
\end{tabular}

All the values need to be multiplied by $l^{3} /$ EI.

It is with only diagonal terms in the mass matrix. By substituting the values in Table 1 into (7), the equation becomes

$$
-\omega^{2}\left[\begin{array}{llll}
a_{11} & a_{12} & a_{13} & a_{14} \\
a_{21} & a_{22} & a_{23} & a_{24} \\
a_{31} & a_{32} & a_{33} & a_{34} \\
a_{41} & a_{42} & a_{43} & a_{44}
\end{array}\right]\left[\begin{array}{cccc}
m_{1} & 0 & 0 & 0 \\
0 & m_{2} & 0 & 0 \\
0 & 0 & m_{3} & 0 \\
0 & 0 & 0 & m_{4}
\end{array}\right]\left\{\begin{array}{l}
A_{1} \\
A_{2} \\
A_{3} \\
A_{4}
\end{array}\right\}+\left[\begin{array}{llll}
1 & 0 & 0 & 0 \\
0 & 1 & 0 & 0 \\
0 & 0 & 1 & 0 \\
0 & 0 & 0 & 1
\end{array}\right]\left\{\begin{array}{l}
A_{1} \\
A_{2} \\
A_{3} \\
A_{4}
\end{array}\right\}=\left\{\begin{array}{l}
0 \\
0 \\
0 \\
0
\end{array}\right\}
$$


which can be further simplified to

$$
\frac{\omega^{2} m l^{3}}{\mathrm{EI}}\left[\begin{array}{cccc}
\frac{343}{6144}-\lambda & \frac{25}{768} & \frac{27}{2048} & \frac{5}{3072} \\
\frac{25}{768} & \frac{125}{6144}-\lambda & \frac{9}{1024} & \frac{7}{6144} \\
\frac{27}{2048} & \frac{9}{1024} & \frac{9}{2048}-\lambda & \frac{1}{1536} \\
\frac{5}{3072} & \frac{7}{6144} & \frac{1}{1536} & \frac{1}{6144}-\lambda
\end{array}\right]\left\{\begin{array}{l}
A_{1} \\
A_{2} \\
A_{3} \\
A_{4}
\end{array}\right\}=\left\{\begin{array}{l}
0 \\
0 \\
0 \\
0
\end{array}\right\},
$$

where $\lambda=E I /\left(m l^{3} \omega^{2}\right)$. Thus, the eigenvalues of the equation can be derived. They are $\lambda_{1}=7.86 \times 10^{-2}, \lambda_{2}=1.86 \times 10^{-3}$, $\lambda_{3}=2.27 \times 10^{-4}$, and $\lambda_{4}=5.03 \times 10^{-5}$. The resonant frequencies of the first 4 modes can be obtained easily from $\lambda$ $=\mathrm{EI} /\left(m l^{3} \omega^{2}\right)$ and they are $\omega_{1}^{2}=12.72 \cdot \mathrm{EI} /\left(m l^{3}\right), \omega_{2}^{2}$ $=537.43 \cdot \mathrm{EI} /\left(\mathrm{ml}^{3}\right), \quad \omega_{3}^{2}=4397.79 \cdot \mathrm{EI} /\left(\mathrm{ml}^{3}\right), \quad$ and $\omega_{4}^{2}$ $=19875.47 \cdot \mathrm{EI} /\left(m l^{3}\right)$.

Other than a fixed number of divisions, the cantilever beam can be divided into any number of segments. As long as the cantilever is divided into enough segments with even length, the model can approximate a continuous model and the numerical results can have required accuracy. The element value of the generalized flexibility matrix can be calculated by

$$
a_{i j}= \begin{cases}\frac{1}{2}\left[(n-j) l_{1}+l_{2}\right]^{2} \times\left\{\frac{2}{3}\left[(n-j) l_{1}+l_{2}\right]+(j-i) l_{1}\right\} \frac{1}{\mathrm{EI}} & i \leq j, \\ \left\{\frac{1}{2} \times \frac{1}{3}(i-j) l_{1} \times\left[(n-i) l_{1}+l_{2}\right]^{2}+\frac{1}{2} \times \frac{2}{3}\left[(n-j) l_{1}+l_{2}\right] \times\left[(n-i) l_{1}+l_{2}\right]^{2}\right\} \frac{1}{\mathrm{EI}} & i>j,\end{cases}
$$

where $l_{1}=l / n$ and $l_{2}=l_{1} / 2$. Such a model can be applied to the calculation procedure for the cantilever beam with attached proof masses at desired locations.

To analyze the resonant frequencies of a cantilever beam with attached masses, the mass matrix needs to be modified. Instead of a diagonal matrix with identical coefficients, the modified mass matrix is the combination of several matrices: the original mass matrix without attached mass denoted with $\mathbf{m}_{\mathbf{o}}$ and the attached mass matrices. Equation (14) is the modified mass matrix with two attached masses $m_{a}$ and $m_{b}$. As shown in Figure 3, the attached masses are considered as being concentrated on the cantilever at the $i$ th station and the $j$ th station. The mass matrix of the characteristic equation $\mathbf{m}$ needs to be changed to the equivalent mass matrix $\mathbf{m}_{\text {eff, which is }}$

$$
\begin{aligned}
& m_{\mathrm{eff}}=m_{o}+m_{a}+m_{b}
\end{aligned}
$$

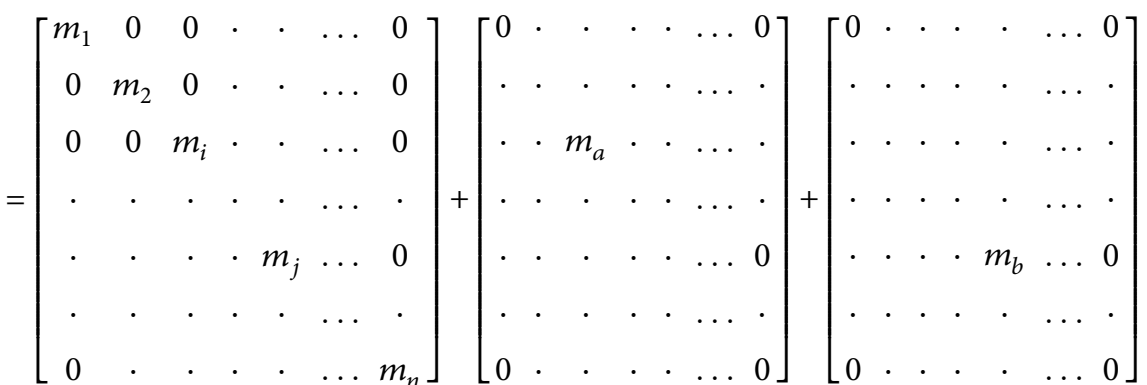

$$
\begin{aligned}
& =\left[\begin{array}{ccccccc}
m_{1} & 0 & 0 & \cdot & \cdot & \ldots & 0 \\
0 & m_{2} & 0 & \cdot & \cdot & \ldots & 0 \\
0 & 0 & m_{i}+m_{a} & \cdot & \ldots & 0 \\
\cdot & \cdot & \cdot & \cdot & \cdot & \ldots & \cdot \\
\cdot & \cdot & \cdot & \cdot & m_{j}+m_{b} & \ldots & 0 \\
\cdot & . & . & . & . & \ldots & \cdot \\
0 & . & . & . & . & \ldots & m_{n}
\end{array}\right]
\end{aligned}
$$




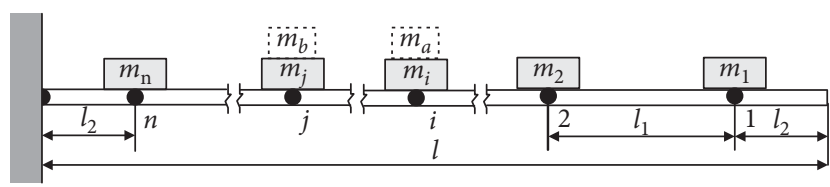

Figure 3: Configuration of a cantilever and attached masses system.

where $\mathbf{m}_{\mathbf{a}}$ and $\mathbf{m}_{\mathbf{b}}$ are mass matrices attached $m_{a}$ and $m_{b}$ at the $i$ th station and the $j$ th station, respectively, $m_{1}=m_{2}=, \ldots,=m_{n}=m / n$. The characteristic equation becomes

$$
-\omega^{2} \cdot \mathbf{a} \cdot \mathbf{m}_{\mathrm{eff}} \cdot \mathbf{A}+\mathbf{I} \cdot \mathbf{A}=\{0\} .
$$

Thus, all the eigenvalues of above matrices can be found by solving the characteristic equation. The corresponding modes' frequencies can be determined accordingly as well.

It is clear that this method can also be applied to inhomogeneous cantilever beams as long as the mass distribution is known and the mass matrix can be synthesized accurately.

\section{Experimental Validation of the Proposed Model by Piezoelectric Cantilever}

3.1. Experimental Setup. To validate the variation of resonant frequency due to attached masses, a piezoelectric cantilever beam that converts deflection caused by mechanical vibration into electricity is used to verify the developed model. The piezoelectric cantilever beam DT2-028K produced by Measurement Specialties Inc. is adopted in this experiment. Two cases of tests are conducted in this study. In the first case, a single proof mass is moving along a cantilever. In the second case, two proof masses are attached on a cantilever, one of the two masses is moving along the cantilever beam while the other is fixed at any position. A $0.3 \mathrm{~g}$ clip and a $0.6 \mathrm{~g}$ clip are used as attached masses in these two cases.

Figure 4 illustrates the physical setup of the experimental platform, which includes a waveform generator, an amplifier, a shaker, a data acquisition device, an oscilloscope, conditioning circuit, and piezoelectric cantilever beam DT2-028K. The properties of DT2-028K are provided by the "Piezo Film Sensors Technical Manual." Its nominal length is $72 \mathrm{~mm}$ as described in the manual. But due to the mounting mechanism installing the cantilever onto the shaker, the effective length of attached cantilever is a bit shorter than its nominal value. The effective length is $70 \mathrm{~mm}$ in this setup. The operating properties of DT2-028K are listed in Table 2. In order to verify the calculated results with actual responses of the cantilever beams, a swept waveform from $1 \mathrm{~Hz}$ to $200 \mathrm{~Hz}$ is generated by the waveform generator. The swept waveform is sent to the amplifier of the shaker and converted to mechanical vibration which induces the deflection of cantilever DT2-028K. Due to the deflection, DT2-028K can generate voltage and current and they are measured by a data acquisition device, PCI-6221, manufactured by National Instrument. The collected data is

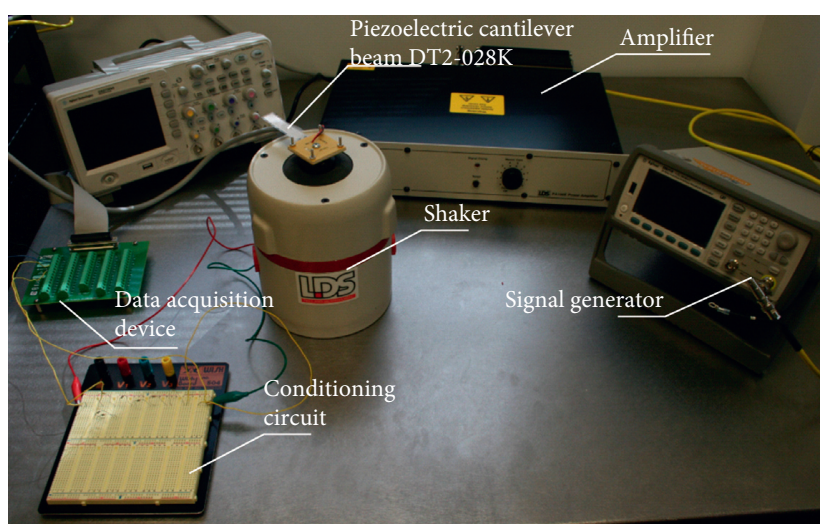

FIGURE 4: Experimental setup of vibration test of a single piezoelectric cantilever beam.

TABLe 2: Properties of piezoelectric cantilever DT2-028K.

\begin{tabular}{lc}
\hline Properties & Value \\
\hline Length $(\mathrm{mm})$ & 72 \\
Effective length $(\mathrm{mm})$ & 70 \\
Width $(\mathrm{mm})$ & 16 \\
Thickness $(\mu \mathrm{m})$ & 230 \\
Density $\left(\mathrm{kg} / \mathrm{m}^{3}\right)$ & 1450 \\
Mass $(\mathrm{g})$ & 0.39 \\
Elasticity modulus $(\mathrm{GPa})$ & 4.2 \\
The first mode frequency $(\mathrm{Hz})$ & 12.9 \\
The second mode frequency $(\mathrm{Hz})$ & 80.9 \\
\hline
\end{tabular}

then processed by MATLAB to verify its frequency response.

Figure 5 illustrates both the frequency responses and the output voltage of DT2-028K without any attached mass. The lower part of this figure is the graph of voltage vs. time. It is apparent that the voltage changes at all times. The value of the voltage depends entirely on the deflection of DT2-028K which changes with the swept waveform. The voltage value is measured by a data acquisition device and then processed by MATLAB to obtain the frequency response (magnitude vs. frequency) as shown in the upper part of Figure 5.

With discrete modelling introduced in Section 2.2, the resonant frequencies of a cantilever beam can be obtained by lumping all the segments into a matrix representation. To achieve an accurate estimation of resonant frequencies in different modes, the number of segments $n$ of the cantilever beam which is one of the important factors needs to be determined. This number is critical since it determines the accuracy of the estimated frequencies value as well as the computation speed. A small number of segments can result in inaccurate estimation and a large value of $n$ yields long calculation time. Thus, the trade-off between the calculation time and the frequency deviation is examined in this study. The frequency deviation is defined as the ratio of the difference between the estimated frequency calculated by discrete model and the actual frequency to the actual frequency, which is 

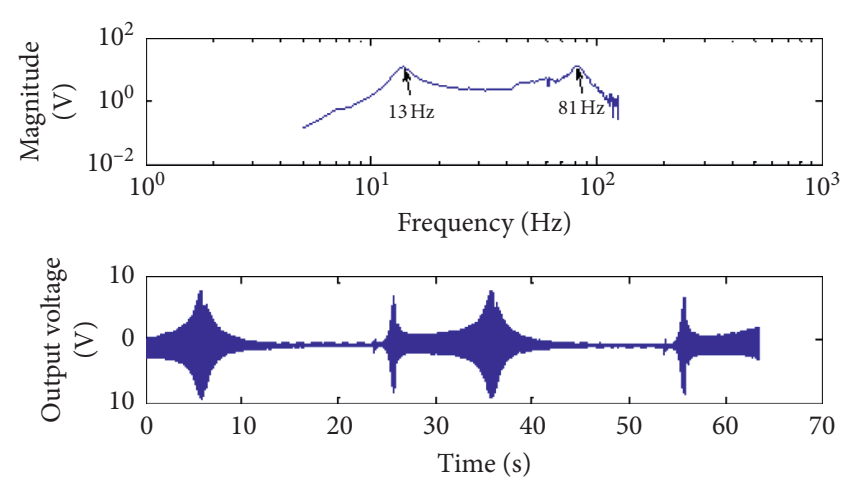

Figure 5: Frequency and time responses of the piezoelectric cantilever beam DT2-028K.

$$
\frac{\left|f_{e}-f_{a}\right|}{f_{a}} \times 100 \%
$$

where $f_{e}$ is the estimated frequency and $f_{a}$ is the actual frequency. In this setup, the actual frequency of DT2-028K is $12.9 \mathrm{~Hz}$ and $80.9 \mathrm{~Hz}$ for the first mode and second mode, respectively, as shown in Table 2. Figure 6 shows the variation of the calculation time and frequency deviation with changes in the number of segments. It is obvious that the increase of calculation time is always accompanied with the growth of the segments number, yet the frequency deviation steadily reduces. It is also clear that the deviation result can converge to a constant as the number of segments increases. As the figure indicated, the curve of mode 1 frequency deviation and the curve of calculation time intersect at the point where the number of segments is about 16 . The calculation time and the mode 1 frequency deviation achieve the trade-off at this point. The trade-off between calculation time and the mode 2 frequency deviation is gotten while the number of segments changes to 18 . Considering both required accuracy and computation load for both mode 1 and mode 2, the number of segments $n$ is suggested to be 18 . Under this condition, the calculation time is about $40 \mathrm{~s}$ and the deviation for mode 1 and mode 2 is $0.16 \%$ and $0.25 \%$ separately.

\subsection{Experimental Validation of the Proposed Model}

3.2.1. Case I: Variation of Resonant Frequencies with a Single Attached Mass. To verify effectiveness of the proposed model, experimental results of single attached mass and dual attached masses are compared. In this case, a proof mass is moved along the cantilever. To simplify the calculation, the attached mass is assumed to be lumped or concentrated. In other words, the attached mass is a point load. Based on the results shown in Figure 6, the cantilever is divided into 18 segments. The attached mass is placed on the centre of each segment, individually, and the configuration of each segment is similar to the configuration shown in Figure 3. To estimate the adjusted resonant frequencies as the attached mass moving along the cantilever beam, the matrix $\mathbf{a}$ is modified accordingly. From the model developed in the previous section, the estimated resonant frequencies of the first four modes (mode 1 , mode 2 , mode 3 , and mode 4 ) of DT2-028K with a $0.3 \mathrm{~g}$ attached mass are illustrated in Figure 7. For mode 1, the resonant frequency drops as the attached mass moves to the tip of the free end of the cantilever beam. The resonant frequencies of modes 2, 3, and 4 can be increased or decreased depending on the locations of the attached mass.

Due to the limitation of the hardware setup, only mode 1 and mode 2 frequencies are verified in this experiment. Mazeika et al. [11] investigate in their research that frequencies of mode 1 and mode 2 are more essential than other higher mode frequencies to be studied. The frequency response in Figure 5 also illustrates the amplitude of output is decayed significantly at higher modes' frequencies. From the view of harvesting energy, it is proved that cantilever can generate much power as operating in mode 2 than mode 1 , and the power produced in mode 2 far outweighs that produced in higher modes.

Figures 8(a) and 8(b) compare the difference of resonant frequencies between theoretical results and experimental results for modes 1 and 2 with attached $0.3 \mathrm{~g}$ and $0.6 \mathrm{~g}$ mass moving along the cantilever separately. From the figure, it is easy to see that the experimental results demonstrate a good match to the theoretical estimation.

The variation range of the frequency for the adopted piezoelectric cantilever devices is also shown in the figure. Reduction of the mode 1 frequency is accompanied with the attached mass moving far away from the boot of the cantilever whether the attached mass is $0.3 \mathrm{~g}$ or $0.6 \mathrm{~g}$. When the attached mass is $0.3 \mathrm{~g}$, frequencies of mode 1 can be tuned to the range from $6 \mathrm{~Hz}$ to $13 \mathrm{~Hz}$, and the range is $50 \mathrm{~Hz}$ to $81 \mathrm{~Hz}$ for mode 2 as shown in Figure 8(a). As the attached mass changes to $0.6 \mathrm{~g}$, the mode 1 and mode 2 frequency can be adjusted in a range from $5 \mathrm{~Hz}$ to $13 \mathrm{~Hz}$ and $42 \mathrm{~Hz}$ to $81 \mathrm{~Hz}$ separately. Thus, the resonant frequencies can be adjusted to be in a desired range with a single attached mass.

\subsubsection{Case II: Variation of Resonant Frequencies with Dual} Attached Masses. In this case, two conditions of vibration modes of the adopted cantilever beam with two attached proof masses are investigated. In the first condition, a $0.3 \mathrm{~g}$ mass is fixed at the middle of the cantilever beam while another $0.3 \mathrm{~g}$ mass is moving along it, as shown in Figure 9. In the second condition, the identical $0.3 \mathrm{~g}$ mass is fixed at the free end of the cantilever beam while another $0.3 \mathrm{~g}$ mass is moving along it, as shown in Figure 10.

Figure 9 also illustrates comparison of the first two modes between theoretical results and experiment results of DT2-028K with a $0.3 \mathrm{~g}$ mass fixed at the middle of the cantilever beam while another $0.3 \mathrm{~g}$ mass is moving along it. The first mode frequency changes from $6 \mathrm{~Hz}$ to $11 \mathrm{~Hz}$ and the second mode frequency varies from $40 \mathrm{~Hz}$ to $55 \mathrm{~Hz}$. Figure 10 demonstrates the results when a $0.3 \mathrm{~g}$ mass is placed at the end of DT2-028K and another same mass is moving along the cantilever. As the figure shows, the first mode frequency changes from $4.9 \mathrm{~Hz}$ to $6.4 \mathrm{~Hz}$ and the second mode frequency varies from $38 \mathrm{~Hz}$ to $64 \mathrm{~Hz}$. Both 


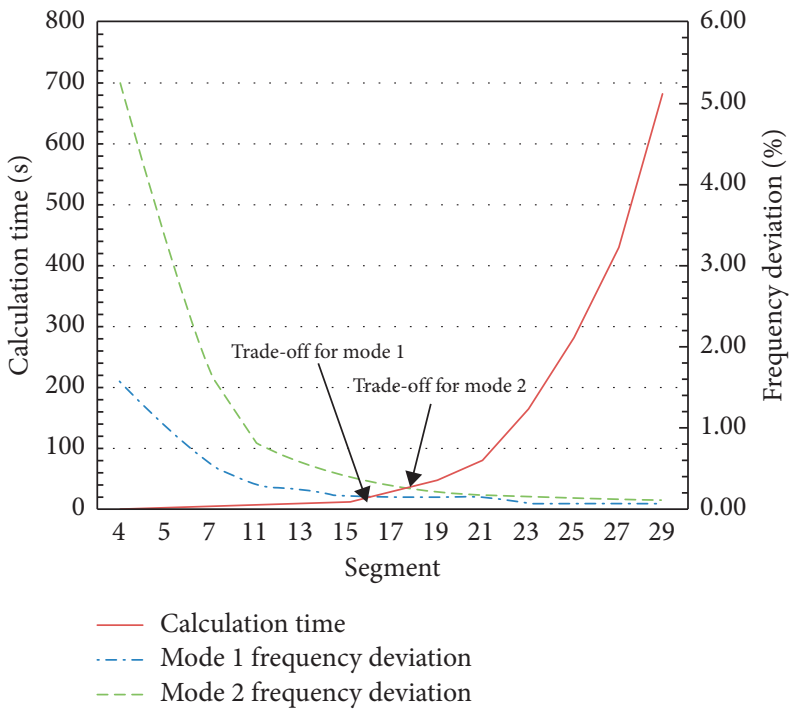

Figure 6: The trade-off between calculation time and frequency deviation.

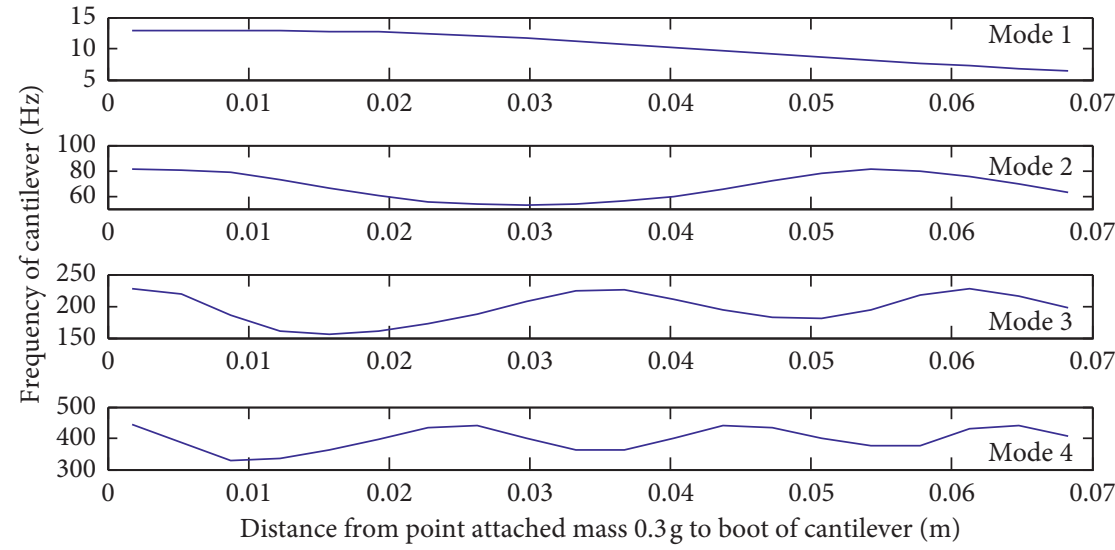

Figure 7: Model estimation of the first four modes' resonant frequencies for DT2-028K.

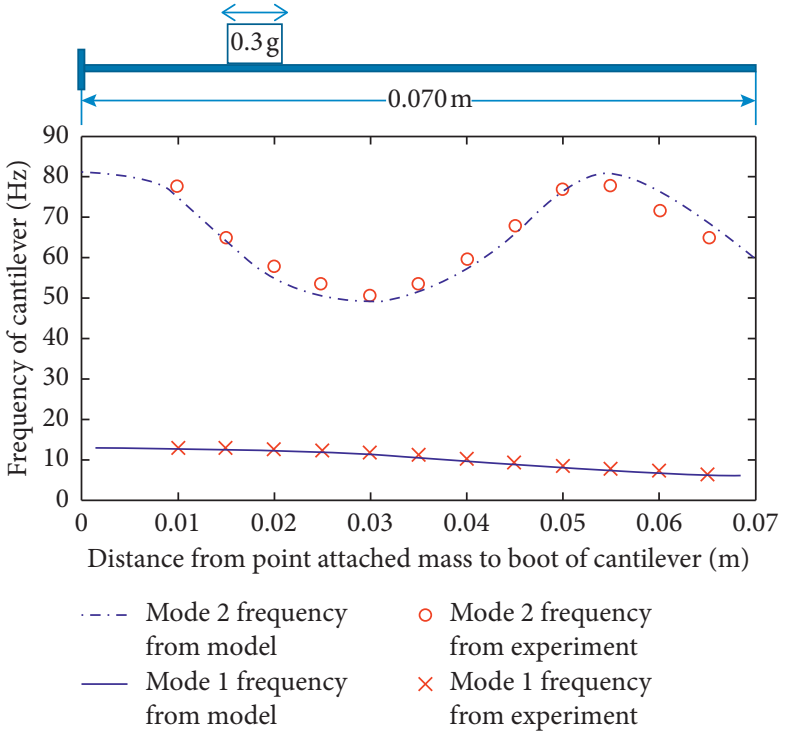

(a)

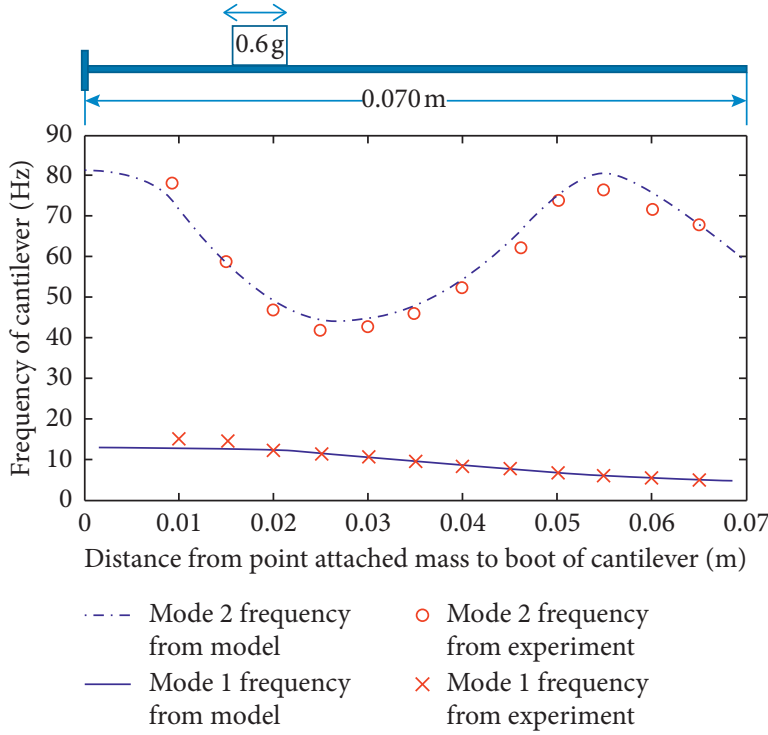

(b)

FIGURE 8: Modes frequencies comparison between theory and experiment for DT2-028K with single attached mass. (a) $0.3 \mathrm{~g}$ attached mass. (b) $0.6 \mathrm{~g}$ attached mass. 

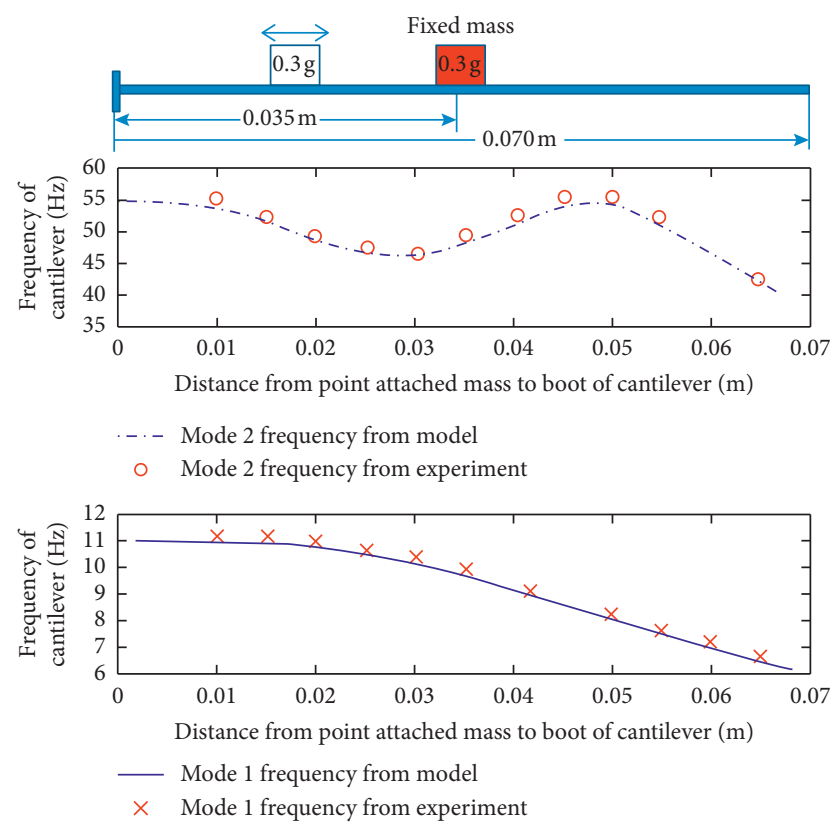

Figure 9: Modes' frequencies comparison between theory and experiment for DT2-028K with a $0.3 \mathrm{~g}$ mass fixed at the middle.
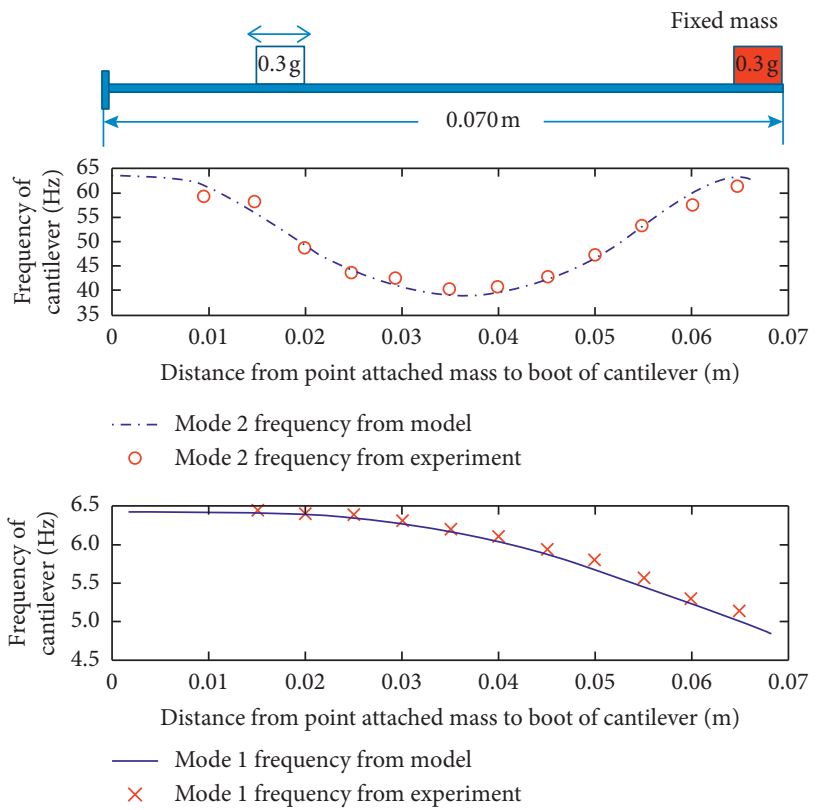

Figure 10: Modes' frequencies comparison between theory and experiment for DT2-028K with a $0.3 \mathrm{~g}$ mass fixed at the end.

figures show that the experiment results can accurately match the theoretical results. From the results, it is can be seen that the applicable range of frequencies of the cantilever beam can be quite different while the fixed attached masses are placed at different positions, though the total weight of attached masses is same. For instance, the first mode has wider variation range while the fixed mass is attached at the middle of the cantilever beam. On the contrary, it provides wider variation range in the second mode as the fixed mass is attached at the free end of the cantilever beam. Although the movable mass travels the same distance, the sensitivity of frequency can be varied.

3.3. Fine-Tuning of Frequencies with Dual Attached Masses. To fine-tune the resonant frequencies, multiple attached masses can be used to make the applicable frequency change in a desired range. It is sometimes difficult to place a mass at certain locations on the cantilever beam in practical applications. Thus, it can be an effective way to fine-tune the resonant frequencies to the desired value with two or more attached masses. This proposed discrete model provides such a tool to estimate the ranges of tuned frequencies for such a scenario by adjusting the mass matrix. In this study, different conditions of vibration modes of the adopted cantilever DT2-028K attached a single mass of $0.6 \mathrm{~g}$ and two same masses of $0.3 \mathrm{~g}$, respectively, are investigated. With the total attached mass $0.6 \mathrm{~g}$, the variation range of resonant frequencies can be overlapped depending on how the attached masses are distributed. Three scenarios are studied:

(a) A single $0.6 \mathrm{~g}$ mass moves along the cantilever beam

(b) One $0.3 \mathrm{~g}$ mass is located at the middle of the cantilever while another $0.3 \mathrm{~g}$ mass moves along the beam

(c) One $0.3 \mathrm{~g}$ mass is located at the free end of the cantilever while another $0.3 \mathrm{~g}$ mass moves along the beam

Figure 11 illustrates the variation of the mode 1 resonant frequencies while Figure 12 demonstrates the mode 2 . In both figures, curve "a" represents resonant frequencies of a single $0.6 \mathrm{~g}$ mass, curve " $\mathrm{b}$ " illustrates the variation of frequency with a $0.3 \mathrm{~g}$ mass fixed in the middle of the beam as another $0.3 \mathrm{~g}$ mass moves along the beam, and curve " $c$ " demonstrates the results with a $0.3 \mathrm{~g}$ mass fixed at the free end when another $0.3 \mathrm{~g}$ mass moves along the beam. It is expected that the three curves intersect as the position of the attached masses changes. The simulated results clearly demonstrate the same frequencies at the middle and the end of the cantilever for the three scenarios. As Figure 11 shows, the first mode frequency band in scenario "a" is from $5 \mathrm{~Hz}$ to $13 \mathrm{~Hz}$ as it is from $5 \mathrm{~Hz}$ to $6.5 \mathrm{~Hz}$ in scenario "c." It is clear that a wider frequency band is available in " $a$," and " $c$ " is suitable for the work of fine-tuning of frequency because frequency change is not extremely sensitive to the location of the movable mass.

A group of similar curves for the mode 2 frequency are shown in Figure 12, the curve for scenario "a" also presents a wider frequency range, and the range of mode 2 frequency can be adjusted from $45 \mathrm{~Hz}$ to $81 \mathrm{~Hz}$. Comparing with the scenario "a," scenario "b" and scenario "c" illustrate a narrower frequency band. It is from $40 \mathrm{~Hz}$ to $55 \mathrm{~Hz}$ in scenario "b" while it is from $38 \mathrm{~Hz}$ to $64 \mathrm{~Hz}$ in scenario "c." Being different from the results shown in Figure 11, "b" instead of " $c$ " is applicable to fine-tuning the mode 2 frequency. 


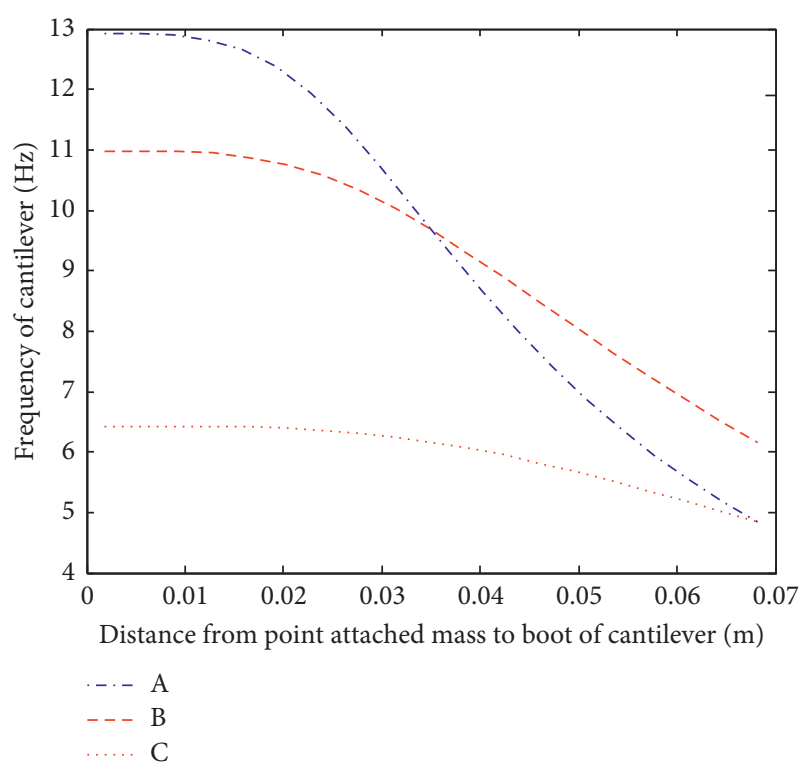

Figure 11: Mode 1 resonant frequency comparison between attaching a $0.6 \mathrm{~g}$ mass and dual mass.

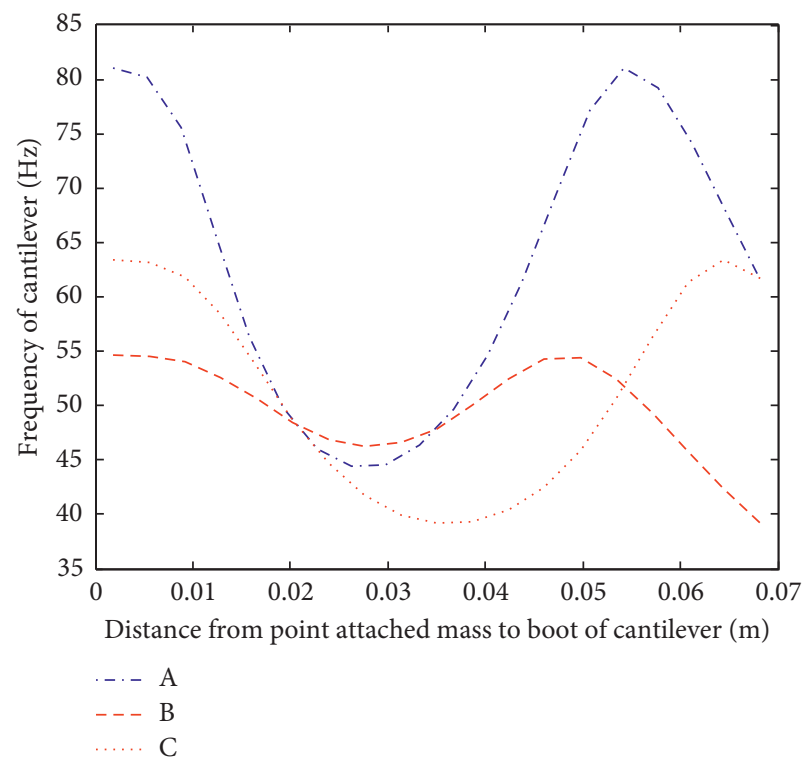

Figure 12: Mode 2 resonant frequency comparison between attaching a $0.6 \mathrm{~g}$ mass and dual mass.

\section{Conclusion}

In this paper, the method of tuning multimodes resonant frequency for piezoelectric cantilever beams using multiple attached masses has been proposed. A discrete-type model to predict and estimate the resonant frequencies of a cantilever beam with multiple attached masses has been also presented. Then an experiment is set up to verify the accuracy of the model and a piezoelectric cantilever beam DT2-028K is adopted in the experiment. The conclusions can be summarized as follows.
The method of using multiple attached masses is effective on adjusting the multimodes' resonant frequency. The amount, the weight, and the location of the attached masses are critical to the effectiveness of the method. When attaching a single $0.3 \mathrm{~g}$ mass, frequencies of mode 1 can be tuned to the range from $6 \mathrm{~Hz}$ to $13 \mathrm{~Hz}$, and the range is $50 \mathrm{~Hz}$ to $81 \mathrm{~Hz}$ for mode 2. As the weight of the attached mass changes to $0.6 \mathrm{~g}$, the mode 1 and mode 2 frequency ranges are adjusted to $5 \mathrm{~Hz}$ to $13 \mathrm{~Hz}$ and $42 \mathrm{~Hz}$ to $81 \mathrm{~Hz}$ separately. Comparing the results of the case attaching a single $0.6 \mathrm{~g}$ mass, the results are very different as attaching dual $0.3 \mathrm{~g}$ masses. With a $0.3 \mathrm{~g}$ mass fixed at the middle of the cantilever beam while another $0.3 \mathrm{~g}$ mass moves along it, the first mode frequency changes from $6 \mathrm{~Hz}$ to $11 \mathrm{~Hz}$ and the second mode frequency varies from $40 \mathrm{~Hz}$ to $55 \mathrm{~Hz}$. When a $0.3 \mathrm{~g}$ mass is placed at the end of DT2-028K and another same mass is moving along the cantilever, the first mode 1 frequency changes from $4.9 \mathrm{~Hz}$ to $6.4 \mathrm{~Hz}$ and the mode 2 frequency varies from $38 \mathrm{~Hz}$ to $64 \mathrm{~Hz}$.

Attaching multiple masses is an effective approach to finetune the resonant frequencies and attaching an individual mass is a good way to widen the resonant frequency range. The mode 2 frequency can be adjusted from $45 \mathrm{~Hz}$ to $81 \mathrm{~Hz}$ using a single $0.6 \mathrm{~g}$ mass moving along the cantilever beam. The range significantly shrinks while using two $0.3 \mathrm{~g}$ masses attached on the cantilever and it is from $40 \mathrm{~Hz}$ to $55 \mathrm{~Hz}$.

The experimental results have also verified the accuracy of the proposed model. The number of segments $n$ of the cantilever beam is critical, since it dominates the accuracy of the estimated frequencies as well as the computation speed. To consider both required accuracy and computation load for both mode 1 and mode 2, the number of segments $n$ is suggested to be 18 in this study. Thus, the calculation time is about $40 \mathrm{~s}$ and the deviation for mode 1 and mode 2 is $0.16 \%$ and $0.25 \%$ separately.

Therefore, the method of using multiple attached masses is a constructive way to adjust the multimodes' resonant frequency which can be settled into the desired range through attaching proof masses. The weight, location, and quantity of attached masses influence frequency bandwidth and sensitivity apparently. Such a model can be a practical and important means to help engineers to adjust resonant frequencies for mechanisms to avoid undesired and utilize desired excitations.

\section{Data Availability}

All data included in this study are available upon request to the corresponding author.

\section{Conflicts of Interest}

The authors declare that they have no conflicts of interest regarding the publication of this paper.

\section{Acknowledgments}

This work was supported in part by the National Key Research and Development Program of China under Grant 
nos. 2018YFE0105300 and 2019YFE0101900, in part by the Postdoctoral Foundation of Beijing Municipal under Grant no. 2017-ZZ-036, in part by the Natural Science Foundation of Tianjin Municipal Science and Technology Commission under Grant no. 17JCYBJC41600, and in part by the National Natural Science Foundation of China under Grant no. 51605010 .

\section{References}

[1] Z. Li, Q. Xu, and L. M. Tam, "Design of a new piezoelectric energy harvesting handrail with vibration and force excitations," IEEE Access, vol. 7, pp. 151449-151458, 2019.

[2] V. Meruane and K. Pichara, "A broadband vibration-based energy harvester using an array of piezoelectric beams connected by springs," Shock and Vibration, vol. 2016, Article ID 9614842, 13 pages, 2016.

[3] M. H. Cheng, Y. Li, E. M. Sabolsky, and C. Chen, "Energy harvesting device with adjustable resonance frequency," ICIC Express Letters, vol. 5, pp. 3315-3320, 2011.

[4] H. A. Sodano, D. J. Inman, and G. Park, "A review of power harvesting from vibration using piezoelectric materials," The Shock and Vibration Digest, vol. 36, no. 3, pp. 197-205, 2004.

[5] X. D. Xie and Q. Wang, "Design of a piezoelectric harvester fixed under the roof of a high-rise building," Engineering Structures, vol. 1179 pages, 2016.

[6] G. Zhou, H. Wang, Z. Zhu, L. Huang, and W. Li, "Performance analysis of wind-induced piezoelectric vibration bimorph cantilever for rotating machinery," Shock and Vibration, vol. 2015, Article ID 216353, 10 pages, 2015.

[7] I. Izadgoshasb, Y. Y. Lim, N. Lake, L. Tang, R. V. Padilla, and T. Kashiwao, "Optimizing orientation of piezoelectric cantilever beam for harvesting energy from human walking," Energy Conversion and Management, vol. 161, pp. 66-73, 2018.

[8] Y. Amini, M. Heshmati, P. Fatehi, and S. E. Habibi, "Piezoelectric energy harvesting from vibrations of a beam subjected to multi-moving loads," Applied Mathematical Modelling, vol. 4916 pages, 2017.

[9] L. Lu, Y. He, T. Wang, T. Shi, and B. Li, "Self-powered wireless sensor for fault diagnosis of wind turbine planetary gearbox," IEEE Access, vol. 7, pp. 87382-87395, 2019.

[10] B. Ju, Z. Guo, Y. Liu, G. Qian, L. Xu, and G. Li, "Self-sensing vibration suppression of piezoelectric cantilever beam based on improved mirror circuit," IEEE Access, vol. 7, pp. 148381-148392, 2019.

[11] D. Mažeika, A. Čeponis, and Y. Yang, "Multifrequency piezoelectric energy harvester based on polygon-shaped cantilever array," Shock and Vibration, vol. 2018, Article ID 5037187, 11 pages, 2018.

[12] S. Priya, "Criterion for material selection in design of bulk piezoelectric energy harvesters," IEEE Transactions on Ultrasonics, Ferroelectrics and Frequency Control, vol. 57, no. 12, pp. 2610-2612, 2010.

[13] R. Hosseini and M. Hamedi, "An investigation into resonant frequency of trapezoidal V-shaped cantilever piezoelectric energy harvester," Microsystem Technologies, vol. 22, no. 5, pp. 1127-1134, 2016.

[14] Y. Hu, H. Hu, and J. Yang, "A low frequency piezoelectric power harvester using a spiral-shaped bimorph," Science in China Series G: Physics, Mechanics and Astronomy, vol. 49, no. 6, pp. 649-659, 2006.

[15] I.-H. Kim, S.-J. Jang, and H.-J. Jung, "Design and experimental study of an L shape piezoelectric energy harvester,"
Shock and Vibration, vol. 2017, Article ID 8523218, 8 pages, 2017.

[16] W. G. Li, S. Shudong Yu, and S. Yu, "Improving power density of a cantilever piezoelectric power harvester through a curved L-shaped proof mass," IEEE Transactions on Industrial Electronics, vol. 57, no. 3, pp. 868-876, 2010.

[17] M. I. Friswell and S. Adhikari, "Sensor shape design for piezoelectric cantilever beams to harvest vibration energy," Journal of Applied Physics, vol. 108, no. 1, pp. 149011-149016, 2010.

[18] D. Avirovik, M. A. Karami, D. Inman, and S. Priya, "L-shaped piezoelectric motor-part II: analytical modeling," IEEE Transactions on Ultrasonics, Ferroelectrics and Frequency Control, vol. 59, no. 1, pp. 108-120, 2012.

[19] Z. Zhang, J. Kan, S. Wang, H. Wang, C. Yang, and S. Chen, "Performance dependence on initial free-end levitation of a magnetically levitated piezoelectric vibration energy harvester with a composite cantilever beam," IEEE Access, vol. 5, pp. 27563-27572, 2017.

[20] T.-K. Chung, C.-M. Wang, P.-C. Yeh, T.-W. Liu, C.-Y. Tseng, and C.-C. Chen, "A three-axial frequency-tunable piezoelectric energy harvester using a magnetic-force configuration," IEEE Sensors Journal, vol. 14, no. 9, pp. 3152-3163, 2014.

[21] M. Li, Y. Wen, P. Li, and J. Yang, "A magnetostrictive/piezoelectric laminate transducer based vibration energy harvester with resonance frequency tunability," in Proceedings of the Sensors IEEE, pp. 1768-1771, IEEE, Limerick, Ireland, October 2011.

[22] M. Rosa and C. De Marqui Junior, "Modeling and analysis of a piezoelectric energy harvester with varying cross-sectional area," Shock and Vibration, vol. 2014, Article ID 930503, 9 pages, 2014.

[23] M. M.M. Defosseux and S. Basrour, "MEMS vibration energy harvesting devices with passive resonance frequency adaptation capability," Journal of Microelectromechanical Systems, vol. 18, no. 6, pp. 1444-1453, 2009.

[24] Ö. Kayac1k, "Integral equation approach for piezo patch vibration control of beams with various types of damping," Computers \& Structures, vol. 86, no. 3, pp. 357-366, 2008.

[25] I. Kucuk, I. S. Sadek, E. Zeini, and S. Adali, "Optimal vibration control of piezolaminated smart beams by the maximum principle," Computers \& Structures, vol. 89, no. 9-10, pp. 744-749, 2011.

[26] H. Ji, J. Qiu, L. Cheng, and H. Nie, "Semi-active vibration control based on unsymmetrical synchronized switch damping: analysis and experimental validation of control performance," Journal of Sound and Vibration, vol. 37022 pages, 2016.

[27] H. X.Y. Hu and Q. Wang, "Broadband piezoelectric energy harvesting devices using multiple bimorphs with different operating frequencies," IEEE Transactions on Ultrasonics, Ferroelectrics, and Frequency Control, vol. 55, no. 9, pp. 2104-2108, 2008.

[28] Y. Song, C. H. Yang, S. J. Hwang, J. H. Kim, and T. H. Sung, "Designing a road energy harvester with multiple piezoelectric cantilever beams and a single tip mass," in Proceedings of 2016 IEEE International Conference on Renewable Energy Research and Applications (ICRERA), pp. 449-451, IEEE, Birmingham, UK, November 2016.

[29] M. I. Friswell, S. F. Ali, O. Bilgen, S. Adhikari, A. W. Lees, and G. Litak, "Non-linear piezoelectric vibration energy harvesting from a vertical cantilever beam with tip mass," Journal of Intelligent Material Systems and Structures, vol. 23, no. 13, pp. 1505-1521, 2012. 
[30] H. Yang, "Vibration control for a cantilever beam with an eccentric tip mass using a piezoelectric actuator and sensor," The International Journal of Acoustics and Vibration, vol. 22, no. 1, 2017.

[31] R. D. Blevins, Formulas for Natural Frequency and Mode Shape, Krieger Publishing, Malabar, Fl, USA, 2001.

[32] K. H. Low, "Frequencies of beams carrying multiple masses: rayleigh estimation versus eigenanalysis solutions," Journal of Sound and Vibration, vol. 268, no. 4, pp. 843-853, 2003. 